\title{
Taking embryonic stem cell potential to the bank: vitrification of the ICM
}

\author{
David F. Albertini
}

Published online: 18 February 2011

(C) Springer Science+Business Media, LLC 2011

As much as our fascination with the potential of stem cells in the future of biomedicine has captured the fancy of scientists and non-scientists alike, the analysis of the ontogeny of this remarkable cell remains within the purview two well-defined research camps. On the one hand are the embryologists and developmental biologists who continue to wrestle with the problem of lineage specification. Their challenge has been one of a kind: how does a single fertilized ovum give rise to the progenitors of the placenta or the fetus?

At the other end of the spectrum are those who deal with the isolation and characterization of ES cells, usually originating from blastocysts, which can ultimately be propagated, expanded, and manipulated into embryonic germ layer phenotypes capable of deploying their differentiation programs to completion. Here, the challenges have been manifold. Optimizing conditions for inner cell mass isolation and survival in primary culture, sorting out ES cells from the various other cell types that result from blastocyst outgrowths, and maintaining derivative lines under conditions that allow for adequate proliferation and totipotency-each of these representing years of dedicated efforts draw in large measure from pioneering studies made using the mouse model.

Our lead article this month takes a giant step towards harnessing the remarkable birthright of ES cells. Desai and collaborators report on the use of vitrification to store inner cell masses from mouse embryos for future derivation of

Capsule Harnessing stem cell potential by vitrification of isolated inner cell masses opens new doors for banking raw materials.

D. F. Albertini $(\bowtie)$

Molecular Medicine,

Kansas CIty, KS, USA

e-mail: dalbertini@kumc.edu stem cells. Their findings yet again illustrate that departure from traditional methodological approaches can open new doors for improving the efficiency and utilization of ES cells for research and therapeutic applications and, if successful in human embryos, would provide direction for ethically appropriate use of these cells.

As discussed in previous issues of JARG, the day to day repertoire used for isolating and establishing human ES cells takes place on the battlefield of the culture dish. War is waged in due course between inner cell mass and trophectoderm cells, amongst others, competing for survival and dominance in the earliest days of derivation. And whether initiated with frozen blastocysts (most commonly) or deposited into cryo storage after several passages, the human ES cell production line as we know it today is one that typically draws upon the use of slow freeze protocols. What Desai and colleagues alert us to is the potential of vitirification.

Vitrification has emerged as an alternative method for embryo storage, and has been adopted for a number of human ART applications that include gonadal tissue and oocyte cryopreservation. It has yet to have gone mainstream in the field of stem cell biology. Given cost effectiveness, improved cell survival, and day to day applicability in the laboratory setting it seems likely that vitrification will find utility for cryopreservation of many cell types of interest in the stem cell field including those produced by induced pluripotent stem cell technology or derived from sources such as cord blood and Wharton's jelly (mesenchymal stem cells). That it has now been demonstrated as a means for storage of the inner cell mass in mouse embryos raises a number of additional opportunities for human materials ranging from derivation efficiency to the prospect of banking the stem cell potential of supernumerary embryos.

The paper by Desai and colleagues from the Cleveland Clinic explores the problem of the culture dish battlefield 
by testing the effects of various methods for inner cell mass isolation that in and of itself hampers isolation of pure stem cell populations due to the intrinsic heterogeneity occurring during intact blastocyst outgrowths. They report good survival of ICM following vitrification using either of two methods and most importantly demonstrate successful passaging of cells that retain major stem cell markers. Many challenges remain including validation of stem cell potential using traditional transplantion approaches to monitor expression of all embryonic germ layer cell types. Moreover, the notorious proclivity that ES cell lines show towards genetic instability upon further passaging will require further investigation using both mouse and human blastocysts. And most importantly, the utility of this approach for human materials must be demonstrated.

If vitrification of isolated human ICMs can be accomplished and used to isolate totipotential ES cell lines, then banking of raw materials will become a reality for dealing with the many stored embryos that have clinical or research utility. Moreover, the investigative framework revealed by this study will offer new insights and a nexus for scientists who seek a deeper understanding of the birthright of these remarkable cells or a level of proficiency consonant with the therapeutic management of human disease. 